\title{
ON SOME INFINITE DIMENSIONAL REPRESEN- TATIONS OF SEMI-SIMPLE LIE ALGEBRAS
}

\author{
HIROSHI KIMURA
}

\section{Introduction}

Let $g$ be a semi-simple Lie algebra over an algebraically closed field $K$ of characteristic 0 . For finite dimensional representations of $\mathfrak{g}$, the following important results are known;

1) $H^{1}(g, V)=0$ for any finite dimensional $g$ space $V$. This is equivalent to the complete reducibility of all the finite dimensional representations.

2) Determination of all irreducible representations in connection with their highest weights.

3) Weyl's formula for the character of irreducible representations [9].

4) Kostant's formula for the multiplicity of weights of irreducible representations [6].

5) The law of the decomposition of the tensor product of two irreducible representations [1].

Harish-Chandra [3] studied the infinite dimensional 8 -spaces with dominant vectors, and established 2) and 3 ) for such spaces. The lacking of the complete reducibility 1) necessitates the study of $\operatorname{Ext}_{\mathfrak{g}}^{1}(U, V)$. A. Hattori determined the structure of $H^{1}(g, V)=\operatorname{Ext}_{\mathfrak{g}}^{1}(K, V)$ for an irreducible $g$-space $V$ with a dominant vector [4]. To study the general case we need more. If $U$ is finite dimensional, we have

$$
\operatorname{Ext}_{\mathfrak{g}}^{1}(U, V)=H^{1}\left(\mathrm{~g}, \operatorname{Hom}_{K}(U, V)\right)=H^{1}\left(\mathrm{~g}, U^{*} \otimes V\right)
$$

where $U^{*}$ is the contragredient representation of $U$, so that we are led to the study of $U^{*} \otimes V$, a special case of 5 ). Theorem 1 of $\$ 2$ concerns with the structure of the tensor product. It follows from this theorem together with a generalization of Hattori's result (Theorem 2) that $\operatorname{Ext}_{\mathfrak{g}}^{1}(U, V)=0$ for certain $U$ and $V$ (Theorem 3 of $\S 3$ ). In $\S 4$ we obtain a formula for the multiplicity of weights in case $\mathfrak{g}=\mathfrak{g l}(3, K)$.

Received February 4, 1964. 


\section{Tensor product}

Let $g$ be a semi-simple Lie algebra over an algebraically closed field $K$ of characteristic $0, \mathfrak{b}$ a Cartan subalgebra and $l$ the rank of $g$. Let $\Delta$ be the set of roots associated with $\mathfrak{h},\left\{\alpha_{1}, \ldots, \alpha_{l}\right\}$ the set of the simple positive roots relative to some ordering and $\Delta^{+}$and $\Delta^{-}$the sets of positive and negative roots, respectively. We write

$$
\mathfrak{n}_{+}==\sum_{\alpha \in \Delta^{+}} g_{\alpha}, \mathfrak{n}_{-}=\sum_{\alpha \in \Delta^{+}} g_{-\alpha} \text { and } \rho=\frac{1}{2} \sum_{\alpha \in \Delta^{+}} \alpha,
$$

where $g_{\alpha}$ is the root space corresponding to $\alpha \in \Delta$.

Let $V_{\Lambda}$ be the representation space of $\mathrm{g}$ with a dominant weight $A$ and $M_{\Lambda}$ the set of the weights of $V_{\Lambda}$. $V_{\Lambda}$ has following decomposition into the weight spaces

$$
V_{\Lambda}=\sum_{\mu \in M_{\Lambda}} V_{\Lambda}(\mu), \mu=\Lambda-\sum m_{i}(\mu) \alpha_{i}
$$

where $m_{i}(\mu)$ are non-negative integers.

Let $V_{\lambda}$ be another representation space with the decomposition

$$
V_{\lambda}=\sum_{\nu \in M_{\lambda}} V_{\lambda}(\nu), \nu=\lambda-\sum n_{i}(\nu) \alpha_{i}
$$

where $n_{i}(\nu)$ are non-negative integers. We consider the tensor product of the representation spaces $V_{\Lambda}$ and $V_{\lambda}$.

THEOREM 1. $V=V_{\Lambda} \otimes V_{\lambda}$ has a sequence of 9-suqspaces

$$
(0)=V_{0} \subset V_{1} \subset \cdots \subset V_{n} \subset \cdots
$$

such that i) $\bigcup_{i} V_{i}=V$ ii) for each $i=1,2, \ldots, V_{i+1} / V_{i}$ is a representation space with a dominant vector and iii) the highest weight of $V_{i+1} / V_{i}$ is of the form $\Lambda+\nu_{i}, \nu_{i} \in M_{\lambda}$. If in particular $V_{\lambda}$ is finite dimensional, then the sequence consists of a finite number of terms.

Proof. We can decompose $V$ into the following form

$$
V=\sum_{i} V(\omega), \omega=\Lambda+\lambda-\sum l_{i}(\omega) \alpha_{i},
$$

where $V(\omega)=\sum_{\mu+\nu=\omega} V_{\Lambda}(\mu) \otimes V_{\lambda}(\nu)$ and $l_{i}(\omega), i=1, \ldots, l$, are non-negative integers. Put $\nu_{0}=\lambda$. Next, let $V_{1}$ be the g-subspace of $V$ generated by $v_{\Lambda} \otimes v_{\lambda}^{\prime}$, $v_{\Lambda} \in V_{\Lambda}(\Lambda), v_{\lambda}^{\prime} \in V_{\Lambda}(\lambda)$, and consider the factor space $V / V_{1}=W^{1}$. Let $W^{1}=\sum_{0}$ 
$W^{1}(\omega), \omega=\mu+\nu, \nu \neq \nu_{0}$ or $\mu \neq \Lambda$, be the decomposition of $W^{1}$ into the weight spaces. Let $\Lambda+\nu_{1}=\Lambda+\lambda-\sum n_{i}\left(\nu_{1}\right) \alpha_{i}$ be a maximal weight in $A_{1}=\{\Lambda+\nu$; $\left.\nu \varepsilon M-\left\{\nu_{0}\right\}\right\}$. It is clear that $W^{1}\left(\Lambda+\nu_{1}\right)$ is finite dimensional. Let $\left\{w_{1}^{1}, \ldots, w_{p_{1}}^{1}\right\}$ be a base of $W^{1}\left(\Lambda+\nu_{1}\right)$. Let $U_{1}^{1}$ be the g-subspace of $W^{1}$ generated by $w_{1}^{1}$. Then there is a $g$-subspace $V_{2}$ of $V$ such that $U_{1}^{1}=V_{2} / V_{1}$. Let $U_{2}^{1}$ be the subspace of $V / V_{2}$ generated by $w_{2}^{1}\left(\bmod V_{2}\right)$. Then there exists a subspace $V_{3}$ of $V$ such that $U_{2}^{1}=V_{3} / V_{2}$. Similarly we get subspaces $V_{4}, \ldots, V_{1+p_{1}}$ of $V$. In considering $A_{2}=\left\{\Lambda+\nu ; \nu \in M-\left\{\lambda, \nu_{1}\right\}\right\}$ by the same manner, we get subspaces $V_{1+p_{1}+1}$ $\subset \cdots \subset V_{1+p_{1}+p_{2}}$. By repeating this process we get a sequence of subspaces of $V$.

$$
(0)=V_{0} \subset V_{1} \subset \cdots \subset V_{n} \subset \cdots
$$

It is clear from the construction of the sequence that $V_{i+1} / V_{i}$ has a dominant vector and its highest weight is of the form $\Lambda+\nu, \nu \in M_{\lambda}(i=1,2, \ldots)$. To see $V^{x}=\bigcup_{i} V_{i}$ coincides with $V$, it is sufficient to show $V(\omega) \subset V^{x}$ for all $\omega$. $V(\omega)$ is spanned by following vectors,

$$
e_{-\alpha}^{a} e_{-\beta}^{b} \cdots e_{-\gamma}^{c} v_{\Lambda} \otimes e_{-\alpha^{\prime}}^{a^{\prime}} e_{-\beta^{\prime}}^{b^{\prime}} \cdots e_{-\gamma^{\prime}}^{c^{\prime}} v_{\Lambda}^{\prime}
$$

where $\alpha, \beta, \ldots, \gamma, \alpha^{\prime}, \beta^{\prime}, \ldots, \gamma^{\prime}$ are positive roots, $e_{-x}, e_{-\beta}, \ldots, e_{-\gamma}, e_{-\alpha^{\prime}}$, $\boldsymbol{e}_{-\beta}, \ldots, \boldsymbol{e}_{-r}$, are non-zero vectors corresponding to $\alpha, \beta, \ldots, \gamma, \alpha^{\prime}, \beta^{\prime}, \ldots$, $\gamma^{\prime}$ respectively, $a, b, \ldots, c, a^{\prime}, b^{\prime}, \ldots, c^{\prime}$ are non-negative integers and $A+\lambda$ $-\left(a \alpha+b \beta+\cdots+c \gamma+a^{\prime} \alpha^{\prime}+b^{\prime} \beta^{\prime}+\cdots c^{\prime} \gamma^{\prime}\right)=\omega$.

Case 1. $\omega=\Lambda+\nu, \nu \in M_{\lambda}$. It is clear that $V^{\mathfrak{r}}$ contains such vectors, i.e. $V(\omega) \subset V^{x}$

Case 2. $\lambda-\sum l_{i}(\omega) \alpha_{i} \notin M_{\lambda}$ and there exists $\nu_{j_{2}} \in M_{\lambda}$ such that $\omega>\Lambda+\nu_{j_{2}}$. Let $\nu_{j_{1}} \in M_{\lambda}$ be the minimal weight such that $\Lambda+\nu j_{1}>\omega$.

i) The case $a^{\prime} \alpha^{\prime}+\cdots+c^{\prime} \gamma^{\prime}=\sum n_{i}\left(\nu j_{1}\right) \alpha_{i}$. By assumption on $j_{1}$ we have $e_{-\delta} e_{-\alpha^{\prime}}^{a^{\prime}} e_{-\beta^{\prime}}^{b^{\prime}} \cdots e_{-\gamma^{\prime}}^{c^{\prime}} v_{\lambda}^{\prime}=0$ for $\delta=\alpha, \beta, \ldots, \gamma$. Therefore

$$
\boldsymbol{e}_{-\alpha}^{a} \cdots \boldsymbol{e}_{-\gamma}^{c}\left(v_{\Lambda} \otimes \boldsymbol{e}_{-\alpha^{\prime}}^{a^{\prime}}, \ldots, \boldsymbol{e}_{-\Upsilon^{\prime}}^{c^{\prime}} v_{\Lambda}^{\prime}\right)=e_{-\alpha}^{a} \cdots e_{-\gamma}^{c} v_{\Lambda} \otimes e_{-\alpha^{\prime}}^{a^{\prime}}, \ldots, e_{-\gamma^{\prime}}^{c^{\prime}} v_{\Lambda}^{\prime} .
$$

From this we have $V(\omega) \subset V^{x}$.

ii) The case $j_{1}=0$.

$$
e_{-\alpha}^{a} \cdots e_{-\zeta}^{c}\left(v_{\Lambda} \otimes v_{\lambda}^{\prime}\right)=e_{-\alpha}^{a} \cdots e_{-\zeta}^{c} v_{\Lambda} \otimes v_{\Lambda}^{\prime} .
$$

Therefore $V(\omega) \subset V^{x}$. 
iii) The case $1+\lambda-\left\{(a-1) \alpha+b \beta+\cdots+c \gamma+a^{\prime} \alpha^{\prime}+\cdots+c^{\prime} \gamma^{\prime}\right\} \geqq \Lambda+\nu_{j}$ We may assume that if $\omega_{1} \geqq \Lambda+\nu_{j_{1}}, V\left(\omega_{1}\right) \subset V^{x}$ and that $e_{-\alpha}^{a-1} e_{-\beta}^{b} \cdots e_{-\tau}^{c} v_{\Lambda} \otimes e_{-\alpha}$ $e_{-a^{\prime}}^{a^{\prime}} \cdots \epsilon_{-\gamma^{\prime}}^{c^{\prime}} v_{\lambda}^{\prime} \in V^{x}$. From the equality,

$$
\begin{aligned}
& e_{-\alpha}\left(e_{-\alpha}^{a-1} e_{-\beta}^{b} \cdots e_{-\gamma}^{c} v_{\Lambda} \otimes e_{-\alpha^{\prime}}^{a^{\prime}} \cdots e_{-\gamma}^{c^{\prime}} v_{\lambda}^{\prime}\right) \\
& \quad-e_{-\alpha}^{\alpha-1} e_{-\beta}^{b} \cdots e_{-\gamma}^{c} v_{\Lambda} \otimes e_{-\alpha} e_{-\alpha^{\prime}}^{a^{\prime}} \cdots e_{-\gamma^{\prime}}^{c^{\prime}} v_{\lambda}^{\prime} \\
& =e_{-\alpha}^{a} e_{-\beta}^{b} \cdots e_{-\gamma}^{c} v_{\Lambda} \otimes e_{-\alpha^{\prime}}^{a^{\prime}} \cdots e_{-\gamma}^{c^{\prime}} v_{\lambda}^{\prime},
\end{aligned}
$$

we have $V(\omega) \subset V^{x}$.

iv) The general case. By i), iii) we can assume that $e_{-\alpha}^{x} \cdots e_{-\gamma}^{z} v_{\Lambda} \otimes e_{-\alpha^{\prime}}^{x^{\prime}}$ $\cdots e_{-\gamma^{\prime}}^{z^{\prime}} v_{\lambda}^{\prime} \in V^{x}$ if $x \alpha+\cdots z \gamma$ is smaller than $a \alpha+\cdots+c \gamma$ or $x^{\prime} \alpha^{\prime}+\cdots z^{\prime} \gamma^{\prime}$ is greater than $a^{\prime} \alpha^{\prime}+\cdots+c^{\prime} \gamma^{\prime}$. From the same equality as in iii) we get $V(\omega) \subset V^{x}$.

Case 3. There exists no weight $\nu_{j} \in M_{\curlywedge}$ such that $\omega>\Lambda+\nu_{i}$. Let $\nu_{j_{1}}$ be a minimal weight such that $\Lambda+\nu j_{1}>\omega$.

i) The case $a^{\prime} \alpha^{\prime}+\cdots+c^{\prime} \gamma^{\prime}=\sum n_{i}\left(\nu j_{1}\right) \alpha_{i}$. This case is treated similarly as case $2, \mathrm{i}$ )

ii) The case $\Lambda+\lambda-\left\{(a-1) \alpha+b \beta+\cdots+c \gamma+a^{\prime} \alpha^{\prime}+\cdots+c^{\prime} \gamma^{\prime}\right\} \geqq \Lambda+\nu_{j_{1}}$ By case 1 and $2, V\left(\omega_{1}\right) \subset V^{x}$ for all $\omega_{1} \geqq \Lambda+\nu_{j_{1}}$. Therefore the result comes from the same equality as in case 2 , iii).

iii) The general case. By use of i), ii) the proof is similar to case 2 , iv).

Finally, if $V_{\lambda}$ is finite dimensional, then

$$
\text { the length of the sequence } \leqq \sum_{\nu \in M \lambda} \operatorname{dim} V(\Lambda+\nu)<\infty \text {. }
$$

This completes the proof of Theorem 1 .

\section{3. $\operatorname{Ext}^{1}\left(V_{\lambda} V_{\Lambda}\right)$}

The factor spaces in Theorem 1 are not necessarily irreducible. Therefore we need to extend Hattori's result to such a space.

THEOREM 2. Let $V$ be a representation space of $g$ with a dominant vector and $\lambda$ its highest weight. Then

$$
H^{1}(g, V)=\left\{\begin{array}{l}
K, \lambda=-\alpha_{i} \\
0, \text { otherwise }
\end{array}\right.
$$

Proof. Let $C$ be the Casimir operator of $V$. First we assume that $C \neq 0$. 
Let $v_{\lambda}$ be a dominant vector. We have $C v_{\lambda}=k v_{\lambda}, k \in K$. Therefore $C v=k v$ for all $v \in V$, so $C V=V$. It follows that $H^{n}(g, V)=0, n=1,2, \ldots$, similarly as in the irreducible case. On the other hand if $V$ is irreducible and $H^{1}(g, V) \neq 0$ it was shown by A. Hattori that $\lambda=-\alpha_{i}$ for some $i$ and that $H^{1}(g, V)=K$. But his proof in [4] does not need the irreducibility. This completes the proof of Theorem 2.

THEOREM 3. Let $V_{\lambda}, V_{\Lambda}$ be the representation spaces with dominant weights $\lambda, \Lambda$ respectively, and assume that $V_{\lambda}$ is finite dimensional. If there is no simple root $\alpha_{i}$ such that $\Lambda+\alpha_{i} \in M_{\lambda}$, we have

$$
\operatorname{Ext}_{g}^{1}\left(V_{\lambda}, V_{\Lambda}\right)=0 .
$$

Proof. Let $V_{\lambda}^{*}$ be the contragredient representation space of $V_{\lambda}$. The set of the weights of $V_{\lambda}^{*}$ is $\left\{-\mu ; \mu \in M_{\lambda}\right\}$. By Theorem 1 there exists the following sequence of $g$-subspaces of $V_{\lambda}^{*} \otimes V_{\Lambda}$,

$$
(0)=V_{0} \subset V_{1} \subset \cdots \subset V_{n}=V_{\lambda}^{*} \otimes V_{\Lambda},
$$

such that $V_{i+1} / V_{i}$ has a dominant vector for each $i$ and its highest weight is of the form $A-\mu, \mu \in M_{\lambda}$. By our assumption, $\Lambda-\mu \neq-\alpha_{i}, i=1, \ldots, l$, for any $\mu \in M_{\lambda}$. Hence we have $H^{1}\left(g, V_{i+1} / V_{i}\right)=0$ by Theorem 2 . By the half exactness of $H^{1}$, we have $H^{1}\left(g, V_{\lambda}^{*} \otimes V_{\Lambda}\right)=0$. Namely

$$
\operatorname{Ext}_{\mathrm{g}}^{1}\left(V_{\lambda}, V_{\Lambda}\right) \cong H^{1}\left(\mathrm{~g}, \operatorname{Hom}_{K}\left(V_{\lambda}, V_{\Lambda}\right)\right) \cong H^{1}\left(g, V_{\lambda}^{*} \otimes V_{\Lambda}\right)=0 .
$$

\section{The multiplicity of a weight}

In this section we consider the multiplicity of weights of an irreducible infinite dimensional representation with a dominant vector for $g=\mathfrak{g}(3, K)$.Let $\left\{\alpha_{1}, \alpha_{2}\right\}$ be a fundamental system of the roots with respect to a Cartan subalgebra $\mathfrak{h}$. Then $\mathfrak{h}$ is spanned by $H_{\alpha_{1}}, H_{\alpha_{2}}$ and the roots are $\pm \alpha_{1}, \pm \alpha_{2}$, $\pm\left(\alpha_{1}+\alpha_{2}\right)$. Let $e_{\alpha}$ be a non-zero vector contained in the rost space $g_{\alpha}$ of $\alpha$. Let $W$ be the Weyl group of $g$. $W$ is generated by $S_{1}=S_{\alpha_{1}}$ and $S_{2}=S_{\alpha_{2}}$, where $S_{\alpha_{i}}$ are given by

$$
S_{\alpha_{i}}(\mu)=\mu-2\left(\mu, \alpha_{i}\right) \alpha_{i} /\left(\alpha_{i}, \alpha_{i}\right), \mu \in \mathfrak{K}_{0}
$$

where $\mathfrak{h}_{0}$ is the real vector space spanned by $\Delta$, and $W=\left\{1, S_{1}, S_{2}, S_{2} S_{1}, S_{1} S_{2}\right.$, $\left.S_{1} S_{2} S_{1}=S_{2} S_{1} S_{2}\right\}$. Let $\left\{f_{1}, f_{2}\right\}$ be the dual base to the base $\left\{2 \alpha_{1} /\left(\alpha_{1}, \alpha_{1}\right), 2 \alpha_{2} /\right.$ $\left.\left(\alpha_{2}, \alpha_{2}\right)\right\}$ of $\mathfrak{h}_{0}$, i.e, 


$$
\left.2\left(\alpha_{i}, f_{j}\right) / \alpha_{i}, \alpha_{i}\right)=\delta_{i j}
$$

Let $\lambda=c_{1} f_{1}+c_{2} f_{2}$ be a highest weight, where the $c_{i}$ are the integers and put $\lambda_{s}=\sigma(\lambda+\rho)-\rho$ for $\sigma \in W$, where $\rho=\frac{1}{2} \sum_{\alpha>0} \alpha=f_{1}+f_{2}$. Then

$$
\begin{aligned}
& \lambda_{S_{1}}=\lambda-\left(c_{1}+1\right) \alpha_{1} \\
& \lambda_{S_{2}}=\lambda-\left(c_{2}+1\right) \alpha_{2} \\
& \lambda s_{S_{2} s_{1}}=\lambda-\left(c_{1}+1\right) \alpha_{1}-\left(c_{1}+c_{2}+2\right) \alpha_{2} \\
& \lambda_{S_{1} s_{2}}=\lambda-\left(c_{2}+1\right) \alpha_{2}-\left(c_{1}+c_{2}+2\right) \alpha_{1} \\
& \lambda_{S_{1} S_{2} s_{1}}=\lambda-\left(c_{1}+c_{2}+2\right)\left(\alpha_{1}+\alpha_{2}\right) .
\end{aligned}
$$

Let $U(g)$ be the universal enveloping algebra and $J_{\lambda}$ the left ideal generated by $\mathfrak{n}_{+}$and $H-\lambda(H), H \in \mathfrak{h}$. We study the structure of $U(\mathfrak{g}) / J_{\lambda}=V_{\lambda}$.

Lemma 4.1. Let $V^{\prime}$ be a g-subspace of $V_{\lambda}$ with a dominant vector and $\lambda^{\prime}$ its highest weight. Then $\lambda \geqq \lambda^{\prime}$ and there exists $\sigma \in W$ such that $\lambda^{\prime}=\lambda_{\text {。 }}$.

Proof. The first part is trivial. Let $\chi_{\lambda}$ and $\chi_{\lambda}$, be the characters of $V_{\lambda}$ and $V_{\lambda}$, respectively. By the definition of the character ([8] Expose 18)

$$
\psi_{\lambda}=\psi_{\lambda}
$$

Therefore, by ([8] Exposé 19, Théorèm 1), there is $\sigma \in W$ such that

$$
\sigma(\lambda+\rho)=\lambda^{\prime}+\rho .
$$

This completes the proof of Lemma 4.1.

Lemma 4.2. For any $\lambda_{0}$ such that $\lambda \geq \lambda_{s}$, there is one and only one sub. space $V_{\lambda_{\sigma}}$.

Proof. If $\lambda_{0}=\lambda$, this is trivial. Therefore we may assume that $\lambda>\lambda_{n}$.

(1) The case $c_{1}+1>0, c_{2}+1>0$. For any $\sigma \in W, \sigma \neq 1, \lambda_{\sigma}<\lambda$. For $\lambda_{s}$ we consider the following vectors,

$$
\begin{aligned}
& v_{\lambda_{S_{1}}}=e_{-\alpha_{1}}^{c_{1}+1} \\
& v_{\lambda_{S_{3}}}=e_{-\alpha_{2}}^{c_{2}+1} \\
& v_{\lambda_{S_{2} S_{1}}}=e_{-\alpha_{2}}^{c_{1}+c_{2}+2} e_{-\alpha_{1}}^{c_{1+1}} \\
& v_{\lambda_{S_{1} S_{2}}}=e_{-\alpha_{1}}^{c_{1}+c_{2}+2} e_{-\alpha_{2}}^{c_{2}+1} \\
& v_{\lambda_{S_{1} S_{2} S_{1}}}=\boldsymbol{e}_{-\alpha_{1}}^{c_{2}+1} e_{-\alpha_{2}}^{c_{1}+c_{2}+2} e_{-\alpha_{1}}^{c_{1}+\mathfrak{l}} .
\end{aligned}
$$

We can easily verify the relations 


$$
e_{\alpha_{i}} v_{\lambda_{\sigma}}=0, i=1,2 \text {. }
$$

Therefore

$$
\mathfrak{n}+v_{\lambda_{\sigma}}=0,
$$

so that there exists a subspace $V_{\lambda_{\sigma}}$ with the dominant vector $v_{\lambda_{\sigma}}$.

(2) The case $c_{2}+1<0, c_{1}+c_{2}+2>0$. $\sigma \in W$ such that $\lambda_{\sigma}<\lambda$ are $S_{1}, S_{2} S_{1}$, $S_{1} S_{2} S_{1}$. If $\sigma=S_{1}$ or $S_{2} S_{1}$ we can construct $V_{\lambda_{\sigma}}$ from $v_{\lambda_{\sigma}}$ in (1). If $\sigma=S_{1} S_{2} S_{1}$, in the expression of $v_{\lambda_{\sigma}}$;

$$
\begin{aligned}
v_{\lambda_{\sigma}} & =e_{-\alpha_{1}}^{c_{2}+1} e_{-\alpha_{2}}^{c_{1}+c_{2}+2} e_{-\alpha_{1}}^{c_{1}+1} \\
& =\sum k_{r} e_{-\alpha_{1}}^{c_{1}+c_{2}+2-r} e_{-\alpha_{2}}^{c_{1}+c_{2}+2-r} e_{-\left(\alpha_{1}+\alpha_{2}\right)}^{r}
\end{aligned}
$$

$c_{1}+c_{2}+2-r \geqq 0$ for $k_{r} \neq 0$, because $e_{-\alpha_{2}}^{c_{1}+c_{2}+2} e_{-\alpha_{1}}^{c_{1}+1}$ is a linear combination of elements

$$
e_{-\alpha_{1}}^{c_{1+1-r}} e_{-\alpha_{2}}^{c_{1}+c_{2}+2-r} e_{-\left(\alpha_{1}+\alpha_{2}\right)}^{r}, r=0,1, \ldots, c_{1}+c_{2}+2 .
$$

And we can verify the relations

$$
e_{\alpha_{2}} v_{\lambda_{\sigma}}=0 . \quad i=1,2
$$

Therefore there exists a subspace $V_{\lambda_{0}}$ with the dominant vector $v_{\lambda_{\sigma}}$

(3) The case $c_{1}+1<0, c_{1}+c_{2}+2>0$. The proof is similar to case (2).

(4) The case $c_{1}+1>0, c_{1}+c_{2}+2 \leqq 0$. There is only one element $\sigma=S_{1}$ such that $\lambda_{3}<\lambda$. Therefore there is the subspace $V_{\lambda_{S_{1}}}$ with the dominant vector $v_{\lambda_{S_{1}}}$.

(5) The case $c_{2}+1>0, c_{1}+2_{2}+2 \leqq 0$. There is the subspace $V_{\lambda_{S 2}}$ with the dominant vector $v_{\lambda_{S 2}}$.

(6) The case $c_{1}+1>0, c_{2}+1=0 . \quad \lambda_{3}<\lambda$ for any $\sigma \neq 1, S_{2}$ and $\lambda_{s_{1}}=\lambda_{s_{1}} s_{2}, \lambda_{S_{2} s_{1}}$ $=\lambda_{S_{1} S_{2} s_{1}}$. Then there are the subspaces $V_{\lambda_{S_{1}}}, V_{\lambda_{S_{2} S_{1}}}$ with the dominant vectors $v_{\lambda_{S_{1}}}, v_{\lambda_{S_{2} S_{1}}}$ respectively.

(7) The case $c_{2}+1>0, c_{1}+1=0$. The proof is similar to case (6).

(8) The case $c_{1}+1 \leqq 0, c_{2}+1 \leqq 0$. There is no element $\sigma \in W$ such that $\lambda_{0}<\lambda$.

Next we shall show that $V_{\lambda_{\sigma}}$ is unique for $\lambda_{\sigma}<\lambda$. Write $\lambda_{\sigma}=\lambda-\sum m_{i} \alpha_{i}$ where $m_{i}$ are non-negative integers and

$$
v_{3}=\sum k_{r} e_{-\alpha_{1}}^{m_{1}-r} e_{-\alpha_{2}}^{m_{2}-r} e_{-\left(\alpha_{1}+\alpha_{2}\right)}^{r} \quad \text { where } r \leqq \min \left(m_{1}, m_{2}\right) .
$$

$v_{\sigma}$ is a dominant vector if and only if 


$$
\begin{aligned}
& \boldsymbol{e}_{\alpha_{1}} v_{\sigma}=0 \\
& \boldsymbol{e}_{\alpha_{2}} v_{\sigma}=0 .
\end{aligned}
$$

We can verify that the coefficients $\left\{k_{r}\right\}$ satisfying (i), (ii) are unique for $\sigma$. This completes the proof of Lemma 4.2.

LEMMA 4.3. Let $P(\mu)$ be the number of ways in which $\mu$ may be partitioned into a sum of positive roots. Let $\lambda=c_{1} f_{1}+c_{2} f_{2}$, where $c_{i}$ are integers, be the highest weight of an infinite dimensional representation of $\mathrm{g}$ with a dominant vector and $m_{\lambda_{\sigma}}(\mu)=\operatorname{dim} V_{\lambda_{\sigma}}(\mu)$, where $V_{\lambda_{\sigma}}$ is an irreducible g-space. Then

$$
P(\lambda-\mu)=\sum_{\lambda \sigma \equiv \lambda} m_{\lambda_{\sigma}}(\mu) .
$$

Proof. In the decomposition to the weight spaces

$$
U(g) / J_{\lambda}=\sum_{\mu} V_{\lambda}(\mu)
$$

it is clear that

$$
\operatorname{dim} V_{\lambda}(\mu)=P(\lambda-\mu)
$$

First we shall prove that any subspace $V$ of $V_{\lambda}$ is the union of subspaces with dominant vectors. If $V=V_{\lambda}$, then this is clear. So we assume that $V \neq V_{\lambda}$. Then

$$
V=\sum_{\mu} V \cap V(\mu) \text { and } V \cap V_{\lambda}(\lambda)=0
$$

Put ${ }^{1} V=\bigcup_{\gamma_{\lambda_{0}} \subset V} V_{\lambda_{\sigma}}$. We consider $V /{ }^{1} V=V^{\prime}$. Let $\nu$ be the maximal weight of $V^{\prime}$ and $v(\nu)\left(\bmod { }^{1} V\right)$ a non-zero vector belonging to $\nu$. Let $V_{\nu}^{\prime}$ be the subspace of $V^{\prime}$ generated by $v(\nu)\left(\bmod { }^{1} V\right)$. The characters of $V_{\lambda}$ and $V_{\nu}^{\prime}$ are equal. Therefore there is $\sigma \in W$ such that $\nu=\lambda_{\sigma}$. Write

$$
\begin{array}{ll}
e_{\alpha_{1}} v\left(\lambda_{\sigma}\right)=\sum h_{r}^{1} e_{-\alpha_{1}}^{m_{1}-1-r} e_{-\alpha_{2}}^{m_{2}-r} e_{-\left(\alpha_{1}+\alpha_{2}\right)}^{r} & r \leqq \min \left(m_{1}-1, m_{2}\right), \\
e_{\alpha_{2}} v\left(\lambda_{\sigma}\right)=\sum h_{r}^{2} e_{-\alpha_{1}}^{m_{1}-r} e_{-\alpha_{3}}^{m_{2}-1-r} e_{-\left(\alpha_{1}+\alpha_{2}\right)}^{r} & r \leqq \min \left(m_{1}, m_{2}-1\right) .
\end{array}
$$

Then $h_{r}^{1}=-h_{r}^{2}$ for all $r$. On the other hand if $\lambda_{\sigma}>\lambda_{\tau}$, then by the construction of $v_{\lambda_{\sigma}}$ we have $V_{\lambda_{\sigma}} \supset V_{\lambda_{\tau}}$. Therefore

$$
{ }^{1} V=\bigcup_{\lambda_{0}} V_{\lambda_{\sigma}}
$$

where any $\lambda_{0}$ is a maximal weight in the set $\left\{\lambda_{\tau} ; V_{\lambda_{\tau}} \subset V\right\}$.

(1) The case $c_{2}+1<0, c_{1}+c_{2}+2>0$. In this case $\lambda>\lambda_{S_{1}}>\lambda_{S_{2} S_{1}}$ and $\lambda>$. 
$\lambda_{s_{1} s_{2} s_{1}}>\lambda_{S_{2} s_{1}}$. When ${ }^{1} V=V_{\lambda s_{1}}, V_{\lambda S_{2} s_{1}}$ or $V_{\lambda_{S_{1} S_{2} S_{1}}}$ there is no element $v\left(\lambda_{\sigma}\right)$. If ${ }^{1} V=V_{\lambda s_{1}} \cup V_{\lambda s_{2} s_{1}}$, then the vectors of ${ }^{1} V$ belonging to the weights $\lambda_{s_{2} s_{1}}+\alpha_{1}$, $\lambda_{S_{2} S_{1}}+\alpha_{2}$ are $e_{-\alpha_{1}}^{-1} e_{-\alpha_{2}}^{c_{1}+c_{2}+2} e_{-\alpha_{1}}^{c_{1}+1}, e_{-\alpha_{2}}^{c_{1}+c_{2}+1} e_{-\alpha_{1}}^{c_{1}+1}$ respectively. From this we obtain that $h_{r}^{1} \neq-h_{r}^{2}$ for some $r$ if $h_{r}^{1}, h_{r}^{2}$ are not zero for all $r$. Therefore $v\left(\lambda_{\sigma}\right)=v_{\lambda_{\sigma}}$ and $V={ }^{1} V$.

(2) The case $c_{1}+1<0, c_{1}+c_{2}+2>0$. The proof is similar to case (1).

(3) The case $c_{1}+1>0, c_{1}+c_{2}+2<0$. $\lambda_{0}$ such that $\lambda_{0}<\lambda$ is $\lambda_{s_{1}}$. Therefore the result is trivial.

(4) The case $c_{2}+1>0, c_{1}+c_{2}+2<0$. The proof is similar to the case (3).

(5) The case $c_{1}+1>0, c_{2}+1=0 . \lambda>\lambda_{s_{1}}>\lambda_{s_{3} s_{1}}$. If ${ }^{1} V=V_{\lambda_{1}}$, then

$$
e_{\alpha_{1}} v\left(\lambda_{s_{2} s_{1}}\right)=0
$$

Therefore $v\left(\lambda_{S_{2} S_{1}}\right) \in V_{\lambda s_{2} s_{1}}$

(6) The case $c_{2}+1>0, c_{1}+1=0$. The proof is similar to case (5).

(7) The case $c_{1}+1 \leqq 0, c_{2}+1 \leqq 0$. There is no $\lambda$ such that $\lambda_{0}<\lambda$. Therefore $V_{\lambda}$ is a simple $g$-module.

Now we consider a composition series, and we obtain

$$
P(\lambda-\mu)=\sum_{\lambda \geq \lambda_{\sigma}} m_{\lambda_{\sigma}}(\mu) .
$$

This completes the proof of Lemma 4.3.

Theorem 3. Let $g$ be $3(3, K)$. We assume that $\sigma(\lambda+\rho) \neq \lambda+\rho$ unless $\sigma=1$. Then we have

$$
m_{\lambda}(\mu)=\sum_{\lambda \geqq \lambda_{\sigma}} \operatorname{Sg}(\sigma) P\left(\lambda_{\sigma}-\mu\right) .
$$

Proof. (1) The case $c_{1}+1>0, c_{2}+1>0$. This is Kostant's formula.

(2) The other case. We may easily examine that

$$
\sum_{\lambda \geqq \lambda_{\sigma} \geqq \lambda \tau} \operatorname{Sg}(\sigma)= \begin{cases}1, & \tau=1 \\ 0, & \tau \neq 1\end{cases}
$$

By these relations and Lemma 4.3 , we have

$$
\begin{aligned}
m_{\lambda}(\mu) & =\sum_{\lambda \geq \lambda_{\tau}} m_{\lambda \tau}(\mu) \sum_{\lambda \geqq \lambda_{\sigma} \geqq \lambda_{\tau}} \operatorname{Sg}(\sigma) \\
& =\sum_{\lambda \geqq \lambda_{\sigma}} \operatorname{Sg}(\sigma) \sum_{\lambda \sigma \geqq \lambda_{\tau}} m_{\lambda_{\tau}}(\mu) \\
& =\sum_{\lambda \geqq \lambda_{\sigma}} \operatorname{Sg}(\sigma) P\left(\lambda_{\sigma}-\mu\right) .
\end{aligned}
$$


This completes the proof of Theorem 3.

Finally we consider the case that there is $\sigma(\neq 1) \in W$ such that $\sigma(\lambda+\rho)=$ $\lambda+\rho$. By Lemma 4.3 ,

$$
m_{\lambda}(\mu)=P(\lambda-\mu)-\sum_{\lambda<\lambda_{\sigma}} m_{\lambda_{\sigma}}(\mu) .
$$

(1) The case $c_{1}+1>0, c_{1}+c_{2}+2=0$.

$$
\boldsymbol{m}_{\lambda}(\mu)=P(\lambda-\mu)-P\left(\lambda_{s_{1}}-\mu\right) .
$$

(2) The case $c_{2}+1>0, c_{1}+c_{2}+2=0$.

$$
m_{\lambda}(\mu)=P(\lambda-\mu)-P\left(\lambda_{S_{2}}-\mu\right) .
$$

(3) The case $c_{1}+1>0, c_{2}+1=0$.

$$
m_{\lambda}(\mu)=P(\lambda-\mu)-P\left(\lambda_{S_{1}}-\mu\right) .
$$

(4) The case $c_{1}+1=0, c_{2}+1>0$.

$$
m_{\lambda}(\mu)=P(\lambda-\mu)-P\left(\lambda_{s_{2}}-\mu\right) .
$$

\section{REFERENCES}

[1] R. Brauer, Sur la multiplication des caractéristiques des groupes continus et semisimples, C. R. Acad. Sci. Paris, 204 (1937), 1784-1786.

[2] P. Cartier, On H. Weyl's character formula, Bull. Amer. Math. Soc., 67 (1961), 228-230.

[3] Harish Chandra, On some applications of the universal enveloping algebra of a semisimple Lie algebras, Trans. Amer. Math. Soc., 70 (1951), 28-96.

[4] A. Hattori, On 1-cohomology groups of infinite dimensional representations of semisimple Lie algebras, J. Math. Soc. Japan, 16 (1964), 226-229.

[5] N. Iwahori, Theory of Lie groups (In Japanese), Iwanami-Shoten (1957).

[6] B. Kostant, A formula for the multiplicity of a weight, Trans. Amer. Math. Soc., 93 (1959), 53-73.

[7] Y. Matsushima, Theory of Lie algebras (In Japanese), Kyoritsu-Press (1956).

[8] Séminaire "Sophus Lie", Ecole Normale Supérieure, Paris (1955).

[9] H. Weyl, Über die Darstellungen der halbeinfachen Gruppen durch lineare Transformationen, Math. Z., 24 (1926), 328-395.

Tokyo University of Education 